\title{
Case report of a bifurcated fibular (lateral) collateral ligament: which band is the dominant one?
}

\author{
K. Kurtys ${ }^{1}$, B. Gonera ${ }^{1}$, Ł. Olewnik¹, P. Karauda1 ${ }^{1}$ R. Shane Tubbs ${ }^{3}$ 4, 5 , M. Polguj² \\ ${ }^{1}$ Department of Anatomical Dissection and Donation, Medical University of Lodz, Poland \\ 2Department of Normal and Clinical Anatomy, Medical University of Lodz, Poland \\ ${ }^{3}$ Department of Neurosurgery, Tulane University School of Medicine, New Orleans, LA, United States \\ ${ }^{4}$ Department of Neurosurgery and Ochsner Neuroscience Institute, Ochsner Health System, New Orleans, \\ $L A$, United States \\ ${ }^{5}$ Department of Anatomical Sciences, St. George's University, Grenada
}

[Received: 29 June 2020; Accepted: 10 July 2020; Early publication date: 7 August 2020]

\begin{abstract}
Background: The fibular collateral ligament is a permanent and extracapsular ligament of the knee joint. It is located on the lateral aspect of the knee and extends from the lateral epicondyle of the femur to the lateral surface of the head of the fibula. As one of the main knee joint ligaments it is a stabilizer of the posterolateral corner of the knee and resists varus stress. The case report displays the bifurcated variant of the fibular collateral ligament. The aim of this study is to determine which of those bands should be considered dominant.

Materials and methods: Classical anatomical dissection was performed on the left knee joint. The fibular collateral ligament was thoroughly cleansed around its origin, distal attachments, and course. Appropriate morphometric measurements were collected.
\end{abstract}

Results: A bifurcated variant of the fibular collateral ligament with inverted proportions of its two bands (main and accessory one) constitutes our findings. It originated on the lateral epicondyle of the femur. Then it divided into two bands (A1 and A2). Band A1 inserted to the head of the fibula. A bony attachment of band $A 2$ was located on the lateral aspect of the lateral condyle of the tibia. Conclusions: Although the fibular collateral ligament is a permanent structure it presents morphological variations. It is important to constantly extend morphological knowledge for all scientists concerned in anatomy. (Folia Morphol 2021; 80, 3: 730-734)

Key words: fibular collateral ligament, lateral collateral ligament, knee, knee joint, case report

\section{INTRODUCTION}

The knee joint is considered the biggest and one of the most complex joints in the human body. It consists of various structures including many ligaments, which are divided into two main groups, extracapsular and intracapsular. It is due to these ligaments that the knee joint can maintain proper stabilisation during different movements.

Address for correspondence: Dr. K. Kurtys, Department of Anatomical Dissection and Donation, Medical University of Lodz, ul. Żeligowskiego 7/9, 90-136 Łódź, Poland; tel: + 4842630 4949, +48 42630 0749, e-mail: kurtyskonrad@gmail.com

This article is available in open access under Creative Common Attribution-Non-Commercial-No Derivatives 4.0 International (CC BY-NC-ND 4.0) license, allowing to download articles and share them with others as long as they credit the authors and the publisher, but without permission to change them in any way or use them commercially. 
The fibular collateral ligament $(\mathrm{FCL})$ is one of the main extracapsular knee joint ligaments. It is located within the lateral aspect of the knee and originates, traditionally, on the lateral epicondyle of the femur. It then runs distally and attaches to the lateral surface of the head of the fibula $[3,17,28]$. There is no connection between it and the knee joint capsule [3]. The biomechanical functions of the FCL are well known and the most important of them is to resist varus forces. Additionally, it can preclude excessive posterolateral rotation of the tibia relative to the femur $[8,10,15]$.

Lesions of the $\mathrm{FCL}$ are not rare, especially among athletes [16]. However, isolated FCL injuries seldom occur, so clinicians have to face so-called multi-ligament knee injuries. Co-occurrence of lesions of both cruciate ligaments of the knee, anterior and posterior, and structures of the posterolateral corner of the knee, are most frequent [19]. Those in most danger of $\mathrm{FCL}$ rupture are sportsmen exposed to shifting strains on a fully extended knee [16]. The FCL can tear as a result of hyperextension, direct varus stress, or twisting movement when a foot is fixed on the ground and the athlete rapidly changes direction of movement [14].

Anatomical structures tend to vary and can surprise us with their morphological variations $[12,26$, 27]. Such fluctuations are quite common among ligaments, muscles, and their tendons $[1,6,7,11$, 21-25]. Although scientists know a lot about the morphology of the FCL, it can still present some new features. Therefore, a proper and accurate classification system of the FCL based on morphological variations has been created [23].

This study presents a case of the bifurcated $\mathrm{FCL}$ with inverted size proportions of its two bands. It is important to collect such knowledge for all scientists involved in anatomy.

\section{CASE REPORT}

The cadaver of a 71-year-old female was subjected to routine anatomical dissection at the Department of Normal and Clinical Anatomy, Medical University of Lodz, for the purposes of research and the education of medical students. The knee joint was dissected using standard techniques according to a strictly specified protocol [22, 23].

During dissection of the lateral aspect of the knee joint, a bifurcated FCL was recognised. The whole ligament was thoroughly cleansed around its origin, distal attachments, and course. It originated on the

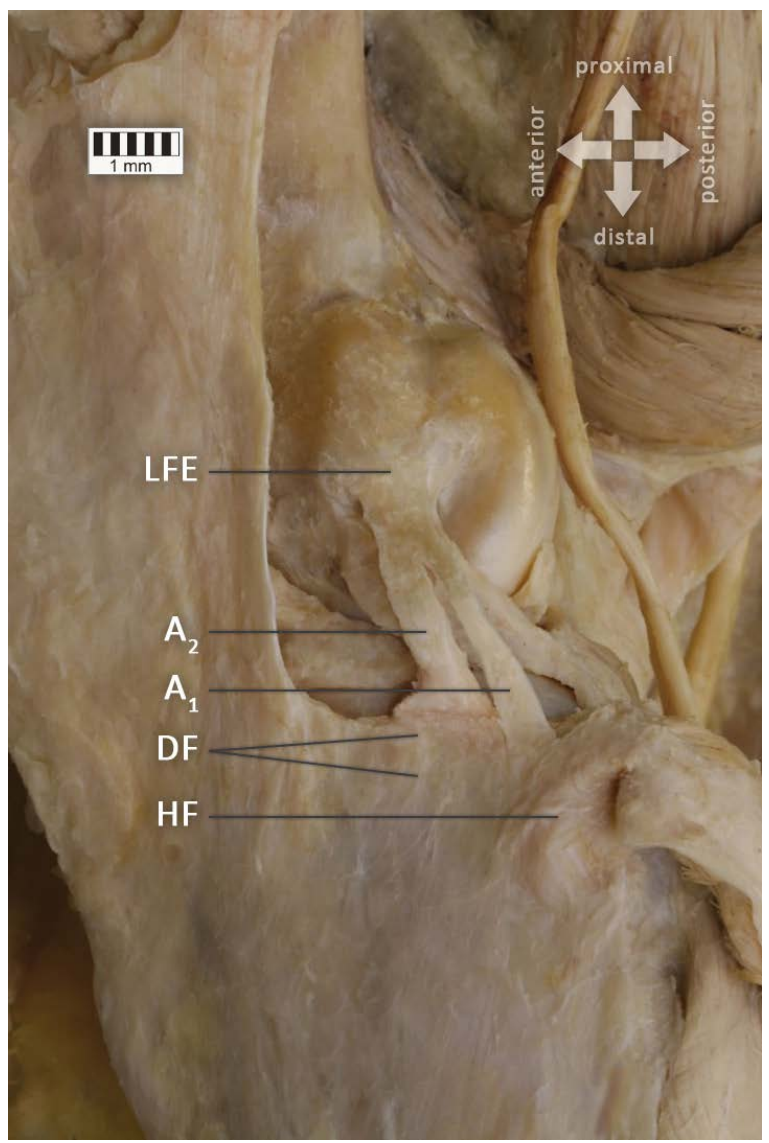

Figure 1. The presented variant of the fibular collateral ligament; a lateral view of the left knee joint; LFE - the lateral femoral epicondyle; $\mathrm{DF}$ - the deep fascia of leg; $\mathrm{HF}$ — the head of the fibula; $A_{1}$ - the main band of the fibular collateral ligament; $A_{2}$ - the accessory band of the fibular collateral ligament.

lateral epicondyle of the femur. Then the common part (band A) divided into two bands ( $\mathrm{A} 1$ and $\mathrm{A} 2$ ). After the cleansing, it was noted that two bands differed in their proportions.

- band A1 ran towards the lateral surface of the head of the fibula and inserted at this point;

- band $A 2$ descended anterior to $A 1$, went under the deep fascia of leg and presented a broad bony attachment on the lateral aspect of the lateral condyle of the tibia.

There were no other morphological abnormalities (Fig. 1).

Appropriate morphometric measurements were acquired. Length and width were taken from digital photographic images and processed through MultiScanBase 18.03 (Computer Scanning System II, Warsaw, Poland), while thickness was measured with an electronic calliper (Mitutoyo Corporation, Kawasaki-shi, Kanagawa, Japan). All results are presented in Table 1. 
Table 1. Measurements of the fibular collateral ligament bands

\begin{tabular}{lcc}
\hline & \multicolumn{2}{c}{ Band } \\
\cline { 2 - 3 } & A1 & A2 \\
\hline Length from the bifurcation point $[\mathrm{mm}]$ & 24.34 & 18.40 \\
Width at the bifurcation point $[\mathrm{mm}]$ & 2.59 & 4.63 \\
Width at the middle point $[\mathrm{mm}]$ & 3.05 & 4.98 \\
Width of the insertion $[\mathrm{mm}]$ & 5.21 & 11.33 \\
Thickness at the bifurcation point $[\mathrm{mm}]$ & 0.32 & 0.41 \\
Thickness at the middle point $[\mathrm{mm}]$ & 0.34 & 0.40 \\
Thickness of the insertion $[\mathrm{mm}]$ & 0.29 & 0.40 \\
\hline
\end{tabular}

\section{DISCUSSION}

Among clinicians, including orthopaedists, there is increasing interest in and hence awareness of anatomical variations. Many morphological classification systems have been published to facilitate better diagnosis and plan more advanced treatment approaches [22-24].

The fibular collateral ligament is a variable structure as demonstrated in some studies. Most of these have focused on abnormalities of its origin around the lateral epicondyle of the femur $[2,5,13,17,29]$. Nevertheless, some have presented morphological variations involving different numbers of FCL bands and points of distal attachments. Specifically, bifurcated and trifurcated FCLs have been recognised [3, 4, 23]. One study even presented a double FCL [23]. According to Olewnik et al. [23], there are four types of FCL (I, Ila, Ilb, III, IV). Among the types that occur as multibands, we can distinguish a main band and one or two accessory band(s). The main band always inserts to the head of the fibula, while the accessory band(s) has/have two points of insertion, the deep fascia of the leg and the styloid process of the fibula. Chappell et al. [3] found all additional bands attached to the anterior or posterior aspect of the head of the fibula. In our study, we recognised two bands, A1 and A2. A1 inserted to the lateral surface of the head of the fibula, while $A 2$ inserted to the lateral aspect of the tibial lateral condyle. The FCL described in this study appeared to be similar to type lla described by Olewnik et al. [23] because of the bifurcation and the descending direction of the bands. However, there was a difference in the attachment point of the accessory band (A2); it presented a bony attachment, while in Olewnik et al. [23] the accessory band inserted to the deep fascia of the leg. On the basis of this information about the insertional points, we could consider band $\mathrm{A} 1$ the main one and band $\mathrm{A} 2$ an accessory one. The question arises whether it is appropriate reasoning in each case and whether the main band is always dominant. In our work, all measurements revealed (Table 1) that the band attached to the lateral aspect of the lateral condyle of the tibia (A2) was more massive than A1. This could imply that despite the atypical "non-main" insertion in such cases, the accessory band can be functionally dominant.

An embryological study found the mesenchymal condensation that provides rise to the $\mathrm{FCL}$ in 7-week-old embryos. At 9 weeks it was described as a thin but well-defined cellular band which descended from the lateral epicondyle of the femur to merge with the perichondrium of the head of the fibula. The FCL reached a structural similarity with the adult knee at 14 weeks, presenting groups of cells separated by a lot of bundles consists of collagenous fibres [9]. According to Merida-Velasco et al. [18], the FCL develops independently from the knee joint capsule in contrast to the medial collateral ligament. It is interesting whether, in the case of bifurcated variants of the $\mathrm{FCL}$, both bands (main and accessory) develop simultaneously.

The FCL is a significant knee joint stabilizer. It resists varus forces at all knee flexion angles and stabilises the posterolateral corner of the knee. Moreover, it resists external rotation of the knee over the flexion span $0^{\circ}-30^{\circ}$. Above $30^{\circ}$ it loses tension and becomes insufficient as a stabilizer of external rotation of the knee $[5,8,10,15,20]$. It is possible that some kinds of multibanded $\mathrm{FCL}$ can provide extra stabilisation functions, but specialist biomechanical examination will be needed to establish this.

Although injuries to the FCL are less common than injuries to the medial collateral ligament, appropriate diagnosis, treatment, and rehabilitation are key to ensuring sufficient recovery and postoperative stabilisation [15, 29]. The treatment method depends on the "grade" of FCL injury, and surgical procedures include primary repair and total reconstruction. Primary repair is used in acute bony avulsions around the proximal or distal attachment of the $\mathrm{FCL}$, while total reconstruction is preferable for midsubstance tears or chronic lateral knee instability after the FCL injury $[19,20]$. Our findings suggest that in some surgical treatment approaches to ligaments or tendons with accessory bands, surgeons should be more careful and verify which band is truly dominant; this should 
be given most focus in order to restore the primary and essential function.

\section{Limitations of the study}

The study has some limitations. Although the anatomical examination was thorough, this is only a case report, presenting the topic for the first time. Further anatomical, biomechanical, and imaging examinations are required to investigate the variation more deeply and create useful tips for clinicians specialising in treatment of ligaments and tendons. Nevertheless, we think it important to collect all new morphological information, and to enrich and integrate anatomical knowledge for all anatomists.

\section{CONCLUSIONS}

The fibular collateral ligament is an anatomical structure with the potential for novel, extremely rare morphological variations. Besides bifurcated, trifurcated, or double FCLs, the size proportions between the main and accessory bands can be inverted. Assembling complete anatomical knowledge, even including such rare cases, is valuable for clinicians involved in anatomy.

\section{Ethics approval and consent to participate}

The protocol of the study was accepted by Bioethics Committee of Medical University of Lodz (resolution RNN/297/17/KE). The cadavers belong to the Department of Normal and Clinical Anatomy of the Medical University of Lodz.

\section{Acknowledgements}

The authors wish to express their gratitude to all those who donated their bodies to medical science.

\section{Conflict of interest: None declared}

\section{REFERENCES}

1. Ahmed SN, Murudkar P, Ahmed M, et al. A morphological study of plantaris muscle and its surgical perspective. Int J Anat Res. 2017; 5(1.3): 3560-3566, doi: 10.16965/ ijar.2016.506.

2. Brinkman JM, Schwering PJA, Blankevoort $L$, et al. The insertion geometry of the posterolateral corner of the knee. J Bone Joint Surg Br. 2005; 87(10): 1364-1368, doi: 10.1302/0301-620X.87B10.16536, indexed in Pubmed: 16189309.

3. Chappell TM, Panchani PN, Moore GD, et al. Morphometry of the fibular collateral ligament: anatomic study with comprehensive review of the literature. Clin Anat. 2014; 27(7): 1089-1096, doi: 10.1002/ca.22416, indexed in Pubmed: 24840861
4. Diamantopoulos A, Tokis A, Tzurbakis M, et al. The posterolateral corner of the knee: evaluation under microsurgical dissection. Arthroscopy. 2005; 21(7): 826-833, doi: 10.1016/j. arthro.2005.03.021, indexed in Pubmed: 16012496.

5. Espregueira-Mendes V, da Silva MV. Anatomy of the lateral collateral ligament: a cadaver and histological study. Knee Surg Sports Traumatol Arthrosc. 2006; 14(3): 221-228, doi: 10.1007/s00167-005-0681-2, indexed in Pubmed: 16220313.

6. Georgiev GP, Landzhov B, Tubbs RS. A novel type of coracobrachialis muscle variation and a proposed new classification. Cureus. 2017; 9(7): e1466, doi: 10.7759/ cureus.1466, indexed in Pubmed: 28936378.

7. Georgiev GP, Tubbs RS, lliev A, et al. Extensor indicis proprius muscle and its variants together with the extensor digitorum brevis manus muscle: a common classification. Clinical significance in hand and reconstructive surgery. Surg Radiol Anat. 2018; 40(3): 271-280, doi: 10.1007/ s00276-018-1981-9, indexed in Pubmed: 29383419.

8. Gollehon DL, Torzilli PA, Warren RF. The role of the posterolateral and cruciate ligaments in the stability of the human knee. A biomechanical study. J Bone Joint Surg. 1987; 69(2): 233-242, doi: 10.2106/00004623198769020-00010.

9. Gray DJ, Gardner E. Prenatal development of the human knee and superior tibiofibular joints. Am J Anat. 1950; 86(2): 235-287, doi: 10.1002/aja.1000860204, indexed in Pubmed: 15410671.

10. Grood ES, Stowers SF, Noyes FR. Limits of movement in the human knee. Effect of sectioning the posterior cruciate ligament and posterolateral structures. J Bone Joint Surg Am. 1988; 70(1): 88-97, indexed in Pubmed: 3335577.

11. Karauda $P$, Olewnik $t$, Podgórski $M$, et al. Anatomical variations of the abductor pollicis longus: a pilot study. Folia Morphol. 2020; 79(4): 817-822, doi: 10.5603/ FM.a2019.0134, indexed in Pubmed: 31802470.

12. Kurtys K, Gonera B, Olewnik $Ł$, et al. A highly complex variant of the plantaris tendon insertion and its potential clinical relevance. Anat Sci Int. 2020; 95(4): 553-558, doi: 10.1007/ s12565-020-00540-4, indexed in Pubmed: 32248353.

13. LaPrade RF, Ly TV, Wentorf FA, et al. The posterolateral attachments of the knee: a qualitative and quantitative morphologic analysis of the fibular collateral ligament, popliteus tendon, popliteofibular ligament, and lateral gastrocnemius tendon. Am J Sports Med. 2003; 31(6): 854-860, doi: 10.1177/03635465030310062101, indexed in Pubmed: 14623649.

14. LaPrade RF, Terry GC. Injuries to the posterolateral aspect of the knee. Association of anatomic injury patterns with clinical instability. Am J Sports Med. 1997; 25(4): 433-438, doi: 10.1177/036354659702500403, indexed in Pubmed: 9240974.

15. LaPrade RF, Tso A, Wentorf FA. Force measurements on the fibular collateral ligament, popliteofibular ligament, and popliteus tendon to applied loads. Am J Sports Med. 2004; 32(7): 1695-1701, doi: 10.1177/0363546503262694, indexed in Pubmed: 15494335.

16. Majewski M, Susanne H, Klaus S. Epidemiology of athletic knee injuries: A 10-year study. Knee. 2006; 13(3): 184-188, doi: 10.1016/j.knee.2006.01.005, indexed in Pubmed: 16603363. 
17. Meister BR, Michael SP, Moyer RA, et al. Anatomy and kinematics of the lateral collateral ligament of the knee. Am J Sports Med. 2000; 28(6): 869-878, doi: 10.1177/03635465000280061601, indexed in Pubmed: 11101111.

18. Mérida-Velasco JA, Sánchez-Montesinos I, Espín-Ferra J, et al. Development of the human knee joint ligaments. Anat Rec. 1997; 248(2): 259-268, doi: 10.1002/(SICI)10970185(199706)248:2<259::AID-AR13>3.0.CO;2-O, indexed in Pubmed: 9185992.

19. Moatshe G, Dean CS, Chahla J, et al. Anatomic fibular collateral ligament reconstruction. Arthrosc Tech. 2016; 5(2): e309-e314, doi: 10.1016/j.eats.2016.01.007, indexed in Pubmed: 27354951.

20. Moulton SG, Matheny LM, James EW, et al. Outcomes following anatomic fibular (lateral) collateral ligament reconstruction. Knee Surg Sports Traumatol Arthrosc. 2015; 23(10): 2960-2966, doi: 10.1007/s00167-015-3634-4, indexed in Pubmed: 25986095.

21. Olewnik $\measuredangle$, Karauda P, Gonera B, et al. Intramuscular innervation of plantaris muscle evaluated using a modified Sihler's staining protocol - Proposal for a new classification. Ann Anat. 2020; 230: 151504, doi: 10.1016/j. aanat.2020.151504, indexed in Pubmed: 32173561.

22. Olewnik $\measuredangle$, Gonera B, Kurtys K, et al. The anterolateral ligament of the knee: a proposed classification system. Clin Anat. 2018; 31(7): 966-973, doi: 10.1002/ca.23267, indexed in Pubmed: 30144325.

23. Olewnik $Ł$, Gonera B, Kurtys K, et al. A proposal for a new classification of the fibular (lateral) collateral ligament based on morphological variations. Ann Anat. 2019;
222: 1-11, doi: 10.1016/j.aanat.2018.10.009, indexed in Pubmed: 30408521.

24. Olewnik $t$, Gonera B, Podgórski M, et al. A proposal for a new classification of pes anserinus morphology. Knee Surg Sports Traumatol Arthrosc. 2019; 27(9): 2984-2993, doi: 10.1007/s00167-018-5318-3, indexed in Pubmed: 30535546 .

25. Olewnik $Ł$, Kurtys K, Gonera B, et al. Proposal for a new classification of plantaris muscle origin and its potential effect on the knee joint. Ann Anat. 2020; 231: 151506, doi: 10.1016/j.aanat.2020.151506, indexed in Pubmed: 32173563.

26. Olewnik $t$, Wysiadecki G, Polguj $M$, et al. The report on the co-occurrence of two different rare anatomic variations of the plantaris muscle tendon on both sides of an individual. Folia Morphol. 2017; 76(2): 331-333, doi: 10.5603/FM.a2016.0069, indexed in Pubmed: 27813626.

27. Olewnik $\measuredangle$, Podgórski $M$, Polguj $M$, et al. The plantaris muscle - rare relations to the neurovascular bundle in the popliteal fossa. Folia Morphol. 2018; 77(4): 785-788, doi: 10.5603/FM.a2018.0039, indexed in Pubmed: 29651792.

28. Recondo JA, Salvador E, Villanúa JA, et al. Lateral stabilizing structures of the knee: functional anatomy and injuries assessed with MR imaging. Radiographics. 2000; 20 (Spec No.): S91-S9S102, doi: 10.1148/radiographics.20.suppl_1. g00oc02s91, indexed in Pubmed: 11046165.

29. Terry GC, LaPrade RF. The posterolateral aspect of the knee. Anatomy and surgical approach. Am J Sports Med. 1996; 24(6): 732-739, doi: 10.1177/036354659602400606, indexed in Pubmed: 8947393. 